\title{
Identification of Polymorphisms in CYP2E1 Gene and Association Analysis among Chronic HBV Patients
}

\author{
Ji-Yong Chun ${ }^{1}$, Byung Lae Park², Hyun Sub \\ Cheong $^{2}$, Jason Y. Kim ${ }^{1}$, Tae Joon Park', Jin \\ Sol Lee, Hyo-Suk Lee ${ }^{3}$, Yoon Jun $\mathrm{Kim}^{3}$ and \\ Hyoung Doo Shin ${ }^{1,2 *}$
}

\begin{abstract}
${ }^{1}$ Department of Life Science, Sogang University, Seoul 121-742, Korea, 'Department of Genetic Epidemiology, SNP Genetics, Inc., Rm 1407, Complex B, WooLim Lion's Valley, Seoul 153-801, Korea, ${ }^{3}$ Department of Internal Medicine and Liver Research Institute, Seoul National University, Seoul 110-744, Korea
\end{abstract}

\begin{abstract}
Cytochrome P450 2E1 (CYP2E1) is a member of the cytochrome P450 superfamily, and it is a key enzyme responsible for the metabolic activation of many smallmolecular-weight compounds such as alcohol, which is classified as a human carcinogen. In this study, we identified 19 single nucleotide polymorphisms (SNPs) in CYP2E1 in Korean population. In these SNPs, we examined possible genetic association of CYP2E1 polymorphisms with HBV clearance and the risk of hepatocellular carcinoma (HCC). Five common polymorphic sites were selected, CYP2E1 polymorphisms at rs3813867, rs3813870, rs2070673, rs2515641 and rs2480257, considering their allele frequencies, haplotype-tagging status and LDs for genotyping in larger-scale subjects $(n=1,092)$. Statistical analysis demonstrated that CYP2E1 polymorphisms and haplotypes show no significant association with HBV clearance, HCC occurrence and onset age of HCC $(p>0.05)$. Previous studies, however, have shown contradictory findings on associations of CYP2E1 polymorphisms with CYP2E1 activities and $\mathrm{HCC}$ risk. Comparing the contrasting results of previous researches suggest that CYP2E1 polymorphism is associated with CYP2E1 activity induced by ethanol, but is not directly associated with HCC risk. CYP2E1 variation/haploype information identified in this study will provide valuable information for future studies on CYP2E1.
\end{abstract}

Keywords: cytochrome P450 2E1 (CYP2E1), hepatocellular carcinoma (HCC), hepatitis B virus (HBV), chronic hepatitis $(\mathrm{CH})$, liver cirrhosis (LC), polymorphism

${ }^{*}$ Corresponding author: E-mail hdshin@sogang.ac.kr Tel +82-2-705-8615, Fax +82-2-2026-4299 Accepted 7 December 2009

\section{Introduction}

The infection of hepatitis virus $B(\mathrm{HBV})$ is considered as an important health problem worldwide with an estimation of 350 million people chronically infected with the disease (Lavanchy, 2005). Although hepatic complications do not develop in most chronic hepatitis B carriers, $15 \%$ to $40 \%$ will develop liver cirrhosis (LC) and hepatocellular carcinoma ( $\mathrm{HCC})$, which come with serious sequelae during their lifetime (Bosch et al., 2005).

Continuous consumption of more than $80 \mathrm{~g}$ of ethanol everyday for a period longer than 10 years increases the risk of HCC by approximately fivefold; however, consumption of less than $80 \mathrm{~g}$ of alcohol per day does not significantly increases the risk of HCC (Morgan et al, 2004). Although the development of alcohol related to HCC involves several factors including the presence of cirrhosis, oxidative stress, disturbed DNA methylation, and defective retinoic acid signaling, the precise mechanisms on how alcohol causes HCC at a molecular level are just beginning to emerge (Seitz \& Stickel 2007; Stickel et al., 2002). According to the International Agency for Research on Cancer, ethanol is classified as a human carcinogen because it induces HCC in animals and increases the risk for developing $\mathrm{HCC}$ in humans (Baan et al., 2007; Seitz and Stickel 2007).

Cytochrome P450 2E1 (CYP2E1), a member of the cytochrome P450 superfamily is important for the metabolic activation of many low-molecular-weight toxicants such as $\mathrm{N}$-nitrosamines, aniline, vinyl chloride, urethane and alcohol (Guengerich et al., 1991). The participation of CYP2E1 in the ethanol metabolizing process is less important than alcohol dehydrogenase (ADH) and aldehyde dehydrogenase (ALDH). However, continuous ethanol consumption is known to increase the activity of CYP2E1 up to 20 fold, a major constituent of the microsomal ethanol oxidizing system in the liver (Stickel \& Osterreicher 2006; Takahashi et al., 1993). Therefore, alcohol' $s$ metabolizing capacity is increased in heavy drinkers. Moreover, CYP2E1 often catalyze the metabolic activation of various procarcinogens to eventual carcinogens (Guengerich et al., 1991; Koop, 1992).

Genetic variation appears to contribute to interindividual variation in CYP2E1 expression levels and activities (McCarver et al., 1998). Specifically, Rsal polymorphism (CYP2E1*5B) (rs2031920) has been associated with decreased CYP2E1 activity or inducibility (Hayashi et al., 1991; Lucas et al., 1995; Marchand et al., 1999; 
Watanabe et al., 1994). Functional CYP2E1 polymorphisms might therefore influence on susceptibility to cancer development. In one study, common genotypes were associated with risk of HCC (Yu et al., 1995). On the other hand, increased risk of HCC was observed with the rare genotype carriers in another study (Ladero et al., 1996). These studies suggest that variation of CYP2E1 can influence individual's risk of HCC.

Based on these studies, we have formed a hypothesis that polymorphisms of CYP2E1 can affect HCC progression among HBV-infected patients and therefore performed a screening of CYP2E1 to examine its genetic association with HBV clearance and HCC progression.

\section{Methods}

\section{Subjects}

A total number of 1,092 Korean subjects having either present or past evidence of HBV infection were prospectively enrolled from the outpatient clinic of the liver unit or from the Center for Health Promotion of Seoul National University Hospital from January 2001 to August 2003. All the study subjects were of Korean ethnicity. Subjects were classified into two different groups: CC (chronic carrier) and SR (spontaneously recovered), according to serological markers. The CC and SR cohorts consisted of 658 and 434 subjects, respectively, and the CC cohort was composed of 338 $\mathrm{CH} / \mathrm{LC}$ and $320 \mathrm{HCC}$ patients (Table 1). The diagnoses of the CC and SR subjects were established by repeated seropositivity for the hepatitis $B$ surface antigen (HBsAg) (Enzygnost ${ }^{\circledR}$ HBsAg 5.0; Dade Behring, Marburg, Germany) over a six-month period, and for both anti-HBs (Enzygnost ${ }^{\circledR}$ Anti-HBs II; Dade Behring, Marburg, Germany) and anti-HBc (AB-Corek; DiaSorin s.r.I.,
Saluggia, Italy) of the IgG type without HBsAg, respectively. We excluded subjects that were positive only for anti-HBs and not for anti-HBc, and those positive for anti-HCV or anti-HIV (GENEDIA ${ }^{\circledR}$; Greencross Life Science Corp., Yongin-shi, Korea, $\mathrm{HCV}^{\mathrm{R}}$ 3.2; Dong-A Pharmaceutical Co., Seoul, Korea). Subjects whose average alcohol consumption assessed by interview was $>510$ $\mathrm{g} /$ day or average cigarette smoking was $>1$ pack/day were excluded. Patients who had any other types of liver diseases such as autoimmune hepatitis, toxic hepatitis, biliary cirrhosis and Budd-Chiari syndrome were also excluded. No patients in our study had a previous history of immunosuppression or anti-viral treatment.

Informed consent was obtained from each patient, and the Institutional Review Board of Human Research at Seoul National University Hospital approved the study protocol. All the patients in the CC group had been on regular medical follow-up and had been evaluated with serum alpha-fetoprotein level assessment, abdominal ultrasonography, and/or 2-phase spiral liver CT scan more than twice a year to detect early stages of HCC. We also performed abdominal MRI, bone scan, chest CT, brain $\mathrm{MRI}$, brain $\mathrm{CT}$, hepatic angiography or PET scan in some patients according to the clinical decisions. Liver cirrhosis was diagnosed pathologically or by the clinical evidences of portal hypertension such as visible collateral vessels on the abdominal wall, esophageal varices on esophagogastroscopy, palpable splenomegaly and sonographically definite findings of cirrhotic liver or ascites. HCC was diagnosed as described previously (the age of onset was determined by the date of the diagnosis.) (Bruix et al., 2001).

\section{Sequence analysis of the human CYP2E1}

We have sequenced exons, including exon-intron boundaries and their flanking regions, including the promoter

Table 1. Clinical profiles of subjects

\begin{tabular}{lccc}
\hline \multirow{2}{*}{ Profile } & SR & \multicolumn{2}{c}{ CC } \\
\cline { 3 - 4 } & & CH or LC & HCC \\
\hline No. of subjects & 434 & 338 & 320 \\
Age (mean (range)) & $54.5(22 \sim 79)$ & $49.7(22 \sim 81)$ & $58.4(24 \sim 79)$ \\
Sex (male/female) & $243 / 191$ & $274 / 64$ & $273 / 47$ \\
HBeAg (positive rate, \%) & 0.2 & 25.8 & 14.5 \\
HBeAb (positive rate, \%) & 0.9 & 23.5 & 32.3 \\
HBsAg (positive rate, \%) & 0 & 77.9 & 73.7 \\
HBsAb (positive rate, \%) & 100 & 0 & 0 \\
U albumin (positive rate, \%) & 0 & 5.3 & 9.9 \\
U blood (positive rate, \%) & 28.1 & 9.2 & 16.4 \\
\hline
\end{tabular}

SR: spontaneously recovered, CC: chronic carrier, CH: chronic hepatitis, LC: liver cirrhosis, HCC: hepatocellular carcinoma. 
region $(1.5 \mathrm{~kb})$ to discover polymorphisms in 24 Korean unregulated individual DNA samples using the $A B I$ PRISM 3700 DNA analyzer (Applied Biosystems, Foster City, CA). Primer sets for the amplification and sequencing analysis of CYP2E1 were designed based on GenBank sequences (Ref. Genome seq.; NT_008818.16). Sequence variants were verified by chromatograms.

\section{Genoyping with Fluorescence Polarization Detec- tion}

For genotyping of polymorphic sites in our HBV study, amplifying primers and probes were designed for TaqMan (Livak, 1999). One allelic probe was labeled with the FAM dye and the other with the fluorescent VIC dye for TaqMan probes. Information regarding the primers is attached in Table 2. Primer Express (Applied Biosystems, Foster City, CA) was used to design the MGB TaqMan probes. PCRs were run in a TaqMan Universal Master Mix without UNG (Applied Biosystems), with PCR primer concentrations of $900 \mathrm{nM}$ and TaqMan MGB-probe concentrations of $200 \mathrm{nM}$. Reactions were performed in a 384-well format in a total reaction volume of $5 \mathrm{ml}$ using $20 \mathrm{ng}$ of genomic DNA. The plates were then placed in a thermal cycler (PE 9700, Applied Biosystems) and heated at $50^{\circ} \mathrm{C}$ for $2 \mathrm{~min}$ and $95^{\circ} \mathrm{C}$ for $10 \mathrm{~min}$, followed by 40 cycles of $95^{\circ} \mathrm{C}$ for $15 \mathrm{~s}$ and $60^{\circ} \mathrm{C}$ for $1 \mathrm{~min}$ with a final soak at $25^{\circ} \mathrm{C}$. The TaqMan assay plates were transferred to the Prism $7900 \mathrm{HT}$ in-

Table 2. Sequences of amplifying and Taqman probe for CYP2E1 SNP genotyping

\begin{tabular}{lll}
\hline \multicolumn{1}{c}{ rs\# } & & \multicolumn{1}{c}{ Probe sequence } \\
\hline$r s 3813867$ & Forward & GCCAACGCCCCTTCTTG \\
& Reverse & TCATTGGTTGTGCTGCACCTA \\
& VIC & ACACTGCACCTCTCCT \\
& FAM & CACTGCAGCTCTCCT \\
$r s 3813870$ & Forward & TCTCTTCATTCTAACCACACACACAAA \\
& Reverse & ATTATTTCTTCATACAGACCCTCTTCCAC \\
& VIC & CTATGGACTACCTTCATAGAA \\
& FAM & CTATGGACTACCTTCGTAGAA \\
$r s 2070673$ & Forward & GTTGTCTAACCAGTGCCAAAGG \\
& Reverse & TTGCCAACCCATAGTTAAGACGT \\
& VIC & CAGGTCGGTACCTC \\
& FAM & CAGGACGGTACCTC \\
$r s 2515641$ & Forward & AGCCAGAACACTTCCTGAATGAAAA \\
& Reverse & CACCTGTGGAAATGGCTTGAAAT \\
& VIC & CTGTACTTGAACTTTC \\
& FAM & ACTGTACTTAAACTTTC \\
$r s 2480257$ & Forward & GTGTGGAGGACACCCTGAC \\
& Reverse & CAAAGAAAGAATCAGTTGAGAAATCCT \\
& VIC & CTTTCAACAAGATTTCAA \\
& FAM & CTTTCAAACAAGTTTTCAA \\
\hline
\end{tabular}

strument (Applied Biosystems) where the fluorescence intensity in each well of the plate was read. Fluorescence data files from each plate were analyzed by automated allele-calling software (SDS 2.1).

\section{Statistics}

We searched for a spine of strong $\left|D^{\prime}\right|$ and LD coefficient $r^{2}$ between all pairs of biallelic loci (Hedrick \& Kumar, 2001). Linkage disequilibrium (LD) was inferred using the algorithm developed by the Broad Institute(using the program Haploview) (Barrett et al., 2005). Haplotypes of each individual were inferred using the algorithm (PHASE, version 2.0) developed by Stephens et al. (Stephens et al., 2001). Subjects harboring missing genotypes were omitted in the analysis of individual single-nucleotide polymorphisms (SNPs) and haplotypes. The genotyping success rate was $>99 \%$, so it is unlikely that omitting a small number of individuals introduced any bias in the analysis. For analysis of viral clearance as an outcome, logistic regression models were used for calculating odds ratios (95\% confidential interval) and corresponding $\mathrm{p}$-values controlling for age (continuous value) and $\operatorname{sex}($ male $=0$, female $=1$ ) as covariates. HBV genotypes, HBV DNA and Alanine transaminase (ALT) levels have been regarded as important factors influencing HBV clearance and the development of HCC. However, HBV genotype $\mathrm{C}$ is predominant among CCs of the virus in Korea (Bae et al., 2005; Odgerel et al., 2003; Song et al., 2005a; Song et al., 2005b; Yoo et al., 2003), and the HBV DNA and ALT levels were found to be fluctuating during the follow-up for the majority of our HBV cohort. Therefore, logistic models for HBV clearance were adjusted only for age and sex. Statistical power is calculated with PGA (Power for Genetic Association Analyses) matlab application (http://dceg.cancer.gov/bb/tools/pga) (Menashe et al., 2008). PGA is an application specifically designed to calculate statistical power and other values of case-control genetic association studies. For the present study, a co-dominant (1df) model with relative risk 1.3, disease prevalence value $7.1 \%$ (Lee et al., 1998), EDF (Effective Degree of Freedom) 2, and alpha error level $5 \%$ were used to calculate the statistical power of $p$-values for HBV clearance and HCC occurrence.

\section{Results}

In this study, we examined the association of CYP2E1 polymorphisms with persistent HBV infection and HCC occurrence. By direct sequencing of 24 individuals, we identified 19 sequence variants in the CYP2E1: 8 in the promoter, 3 in coding regions of exons (two non-synon- 
A. Map of CYP2E1 (cytochrome P450, family 2, subfamily E, polypeptide 1on) on 10q24.3-qter (14 kb)

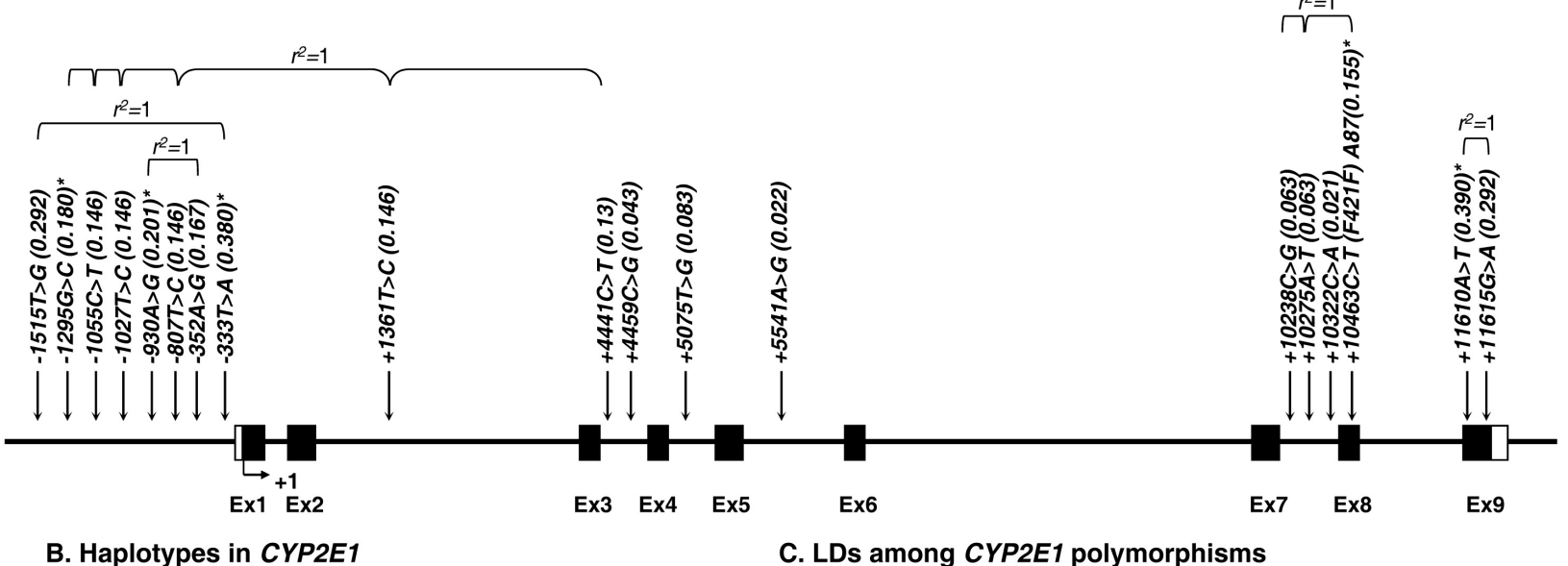

B. Haplotypes in CYP2E1

C. LDs among CYP2E1 polymorphisms
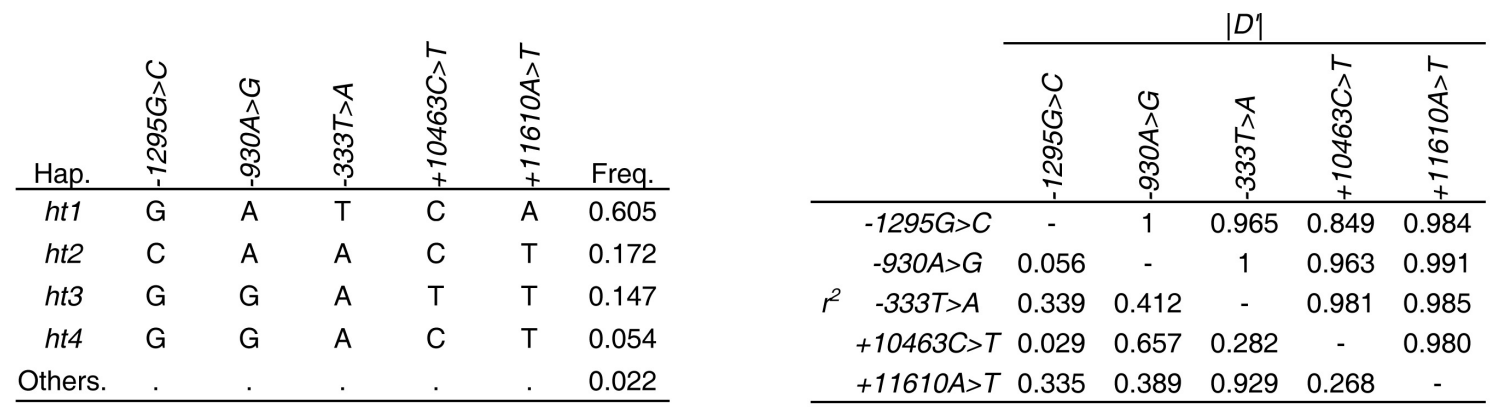

Fig. 1. Gene maps and haplotypes of the CYP2E1. A. Polymorphisms identified in CYP2E1. Coding exons are marked by shaded blocks and 5' and 3'UTR by white blocks. The first base of the translational start site is denoted as nucleotide +1 . Asterisks $\left(^{*}\right)$ indicate polymorphisms genotyped in a larger population $(n=1,092)$. Polymorphisms identified in CYP2E1 on chromosome 10q24.3-qter (Ref. Genome Seq. NT_008818.16). B. Haplotypes of CYP2E1. Only those with frequencies over 0.05 are shown. C. Linkage disequilibrium coefficients $\left(\left|D^{\prime}\right|\right.$ and $\left.r^{2}\right)$ among selected SNPs based on the genotypes of whole study subjects in this study $(n=1,092)$.

ymous and four synonymous) and 8 in introns (Fig. 1A, Table 3). We analyzed five polymorphisms (rs3813867, rs3813870, rs2070673, rs2515641 and rs2480257) in CYP2E1. These were selected for larger-scale genotyping $(n=1,092)$ by considering their allele frequencies, haplotype-tagging status, and LDs. The minor allele frequencies of the SNPs in Korean population were; 0.181 (rs3813867), 0.202 (rs3813879), 0.381 (rs2070673), 0.157 (rs2515641) and 0.392 (rs2480257) (Fig. 1A, Table 3). Genotype distributions of all loci were in Hardy-Weinberg equilibrium ( $p>0.05)$, except for rs3813867 ( $p$ $<0.05$, Table 3). Five SNPs showed low LDs (Fig. 1C), and four major haplotypes accounted for over $97.8 \%$ of the distribution (Fig. 1B).

Association analyses of $\mathrm{HBV}$ clearance $(\mathrm{HCC} / \mathrm{LC} / \mathrm{CH}$ vs. SR) and HCC occurrence (HCC vs. CH/LC) for each polymorphism and haplotype of the CYP2E1 were performed using logistic regression models (Table 4), con- trolling age and sex as covariates (gender and age were significantly associated with HBV clearance; $p<0.05$, data not shown) in our Korean HBV study. The statistical powers for each polymorphism are also shown in Table 4. In the analysis, CYP2E1 polymorphisms and haplotypes did not show significant associations with HBV clearance and the HCC occurrence $(p>0.05$, Table 4). The role of CYP2E1 polymorphisms in onset age of HCC was also analyzed using Cox relative hazards model among chronic HBV patients (Table 4). However, no significant genetic effect was observed afterwards ( $>0.05$, Table 4).

\section{Discussion}

In the present, HCC is one of the most common tumors among the prevalent malignant tumors worldwide (ElSerag \& Mason, 2000). The increase in HCC occurrence 
Table 3. Genotype and allele frequency of polymorphisms in CYP2E1

\begin{tabular}{|c|c|c|c|c|c|c|c|c|c|c|}
\hline \multirow{2}{*}{$\frac{\text { Loci }}{-1515 T>G}$} & \multirow{2}{*}{$\begin{array}{l}\text { Position } \\
\text { Promoter }\end{array}$} & \multirow{2}{*}{ Amino acid change } & \multirow{2}{*}{$\frac{\text { rs\# }}{r s 8192766}$} & \multicolumn{4}{|c|}{ Genotype } & \multirow{2}{*}{$\begin{array}{c}\text { Frequency } \\
0.292\end{array}$} & \multirow{2}{*}{$\frac{\text { Heterozygosity }}{0.413}$} & \multirow{2}{*}{$\frac{\mathrm{HWE}^{1}}{0.344}$} \\
\hline & & & & $\mathrm{T}$ & GT & G & $N$ & & & \\
\hline & & & & 13 & 8 & 3 & 24 & & & \\
\hline \multirow[t]{2}{*}{$-1295 G>C$} & Promoter & & $r s 3813867$ & $\mathrm{G}$ & GC & C & $\mathrm{N}$ & 0.181 & 0.296 & 0.018 \\
\hline & & & & 738 & 298 & 47 & 1,083 & & & \\
\hline \multirow[t]{2}{*}{$-1055 C>T$} & Promoter & & rs2031920 & $C$ & $\mathrm{CT}$ & $\mathrm{T}$ & $\mathrm{N}$ & 0.146 & 0.249 & 0.422 \\
\hline & & & & 18 & 5 & 1 & 24 & & & \\
\hline \multirow[t]{2}{*}{$-1027 T>C$} & Promoter & & rs2031921 & $\mathrm{T}$ & $\mathrm{CT}$ & C & $\mathrm{N}$ & 0.146 & 0.249 & 0.422 \\
\hline & & & & 18 & 5 & 1 & 24 & & & \\
\hline \multirow[t]{2}{*}{$-930 A>G$} & Promoter & & rs3813870 & $A$ & $A G$ & G & $N$ & 0.202 & 0.322 & 0.422 \\
\hline & & & & 686 & 337 & 48 & 1,071 & & & \\
\hline \multirow[t]{2}{*}{$-807 T>C$} & Promoter & & rs2031922 & $\mathrm{T}$ & $\mathrm{CT}$ & $C$ & $\mathrm{~N}$ & 0.146 & 0.249 & 0.422 \\
\hline & & & & 18 & 5 & 1 & 24 & & & \\
\hline \multirow{2}{*}{$-352 A>G$} & Promoter & & rs2070672 & $A$ & $A G$ & G & $\mathrm{N}$ & 0.167 & 0.278 & 0.396 \\
\hline & & & & 12 & 6 & 0 & 18 & & & \\
\hline \multirow[t]{2}{*}{$-333 T>A$} & Promoter & & rs2070673 & $\mathrm{T}$ & TA & A & $\mathrm{N}$ & 0.381 & 0.472 & 0.075 \\
\hline & & & & 427 & 482 & 171 & 1,080 & & & \\
\hline \multirow[t]{2}{*}{$1361 T>C$} & Intron & & rs 943975 & $\mathrm{~T}$ & $\mathrm{CT}$ & $C$ & $N$ & 0.146 & 0.249 & 0.422 \\
\hline & & & & 18 & 5 & 1 & 24 & & & \\
\hline \multirow[t]{2}{*}{$4441 C>T$} & Intron & & rs2070674 & $\mathrm{C}$ & $\mathrm{CT}$ & $\mathrm{T}$ & $\mathrm{N}$ & 0.13 & 0.227 & 0.263 \\
\hline & & & & 18 & 4 & 1 & 23 & & & \\
\hline \multirow[t]{2}{*}{$4459 C>G$} & Intron & & Novel & $C$ & CG & G & $\mathrm{N}$ & 0.043 & 0.083 & 0.827 \\
\hline & & & & 21 & 2 & 0 & 23 & & & \\
\hline \multirow[t]{2}{*}{$5075 T>G$} & Intron & & rs8192773 & $\mathrm{T}$ & GT & G & $\mathrm{N}$ & 0.083 & 0.153 & 0.656 \\
\hline & & & & 20 & 4 & 0 & 24 & & & \\
\hline \multirow[t]{2}{*}{$5541 A>G$} & Intron & & Novel & $A$ & $A G$ & G & $\mathrm{N}$ & 0.022 & 0.043 & 0.915 \\
\hline & & & & 22 & 1 & 0 & 23 & & & \\
\hline \multirow[t]{2}{*}{$10238 C>G$} & Intron & & rs2070676 & $C$ & $\mathrm{CG}$ & G & $\mathrm{N}$ & 0.063 & 0.117 & 0.744 \\
\hline & & & & 21 & 3 & 0 & 24 & & & \\
\hline \multirow[t]{2}{*}{$10275 A>T$} & Intron & & rs2070677 & $A$ & AT & $\mathrm{T}$ & $\mathrm{N}$ & 0.063 & 0.117 & 0.744 \\
\hline & & & & 21 & 3 & 0 & 24 & & & \\
\hline \multirow[t]{2}{*}{$10322 C>A$} & Intron & & Novel & $C$ & $A C$ & A & $\mathrm{N}$ & 0.021 & 0.041 & 0.917 \\
\hline & & & & 23 & 1 & 0 & 24 & & & \\
\hline \multirow[t]{2}{*}{$10463 C>T$} & Cds & Phe421Phe & rs2515641 & $C$ & $\mathrm{CT}$ & $T$ & $\mathrm{~N}$ & 0.157 & 0.265 & 0.561 \\
\hline & & & & 768 & 291 & 24 & 1,083 & & & \\
\hline $11610 A>T$ & 3'utr & & rs2480257 & $A$ & AT & $T$ & $\mathrm{~N}$ & 0.392 & 0.477 & 0.056 \\
\hline & & & & 403 & 471 & 176 & 1,050 & & & \\
\hline $11615 G>A$ & 3'utr & & rs2480256 & $\mathrm{G}$ & $A G$ & A & $N$ & 0.292 & 0.413 & 0.967 \\
\hline & & & & 12 & 10 & 2 & 24 & & & \\
\hline
\end{tabular}

${ }^{1} \mathrm{p}$-values of deviation from Hardy-Weinberg Equilibrium (HWE) among Korean subjects.

Bold face means SNPs genotyped in a larger population $(n=1,092)$ and plain faces were based on the sequencing data $(n=24)$.

is most likely due to the more widespread chronic infection with HBV. The virus-host interactions might influence different disease outcomes of HBV infections (Cruz et al., 1987). However, knowledge of the host genetic factors involved in the progress of HBV infection and HCC occurrence is insufficient now. CYP2E1, an ethanol inducible enzyme, is one of the candidate genes that might influence the outcome of chronic liver disease. Moreover, acetaldehyde, a highly toxic and volatile compound is the first intermediate of alcohol metabolism by CYP2E1 (Peters \& Ward, 1988). Therefore, the level of CYP2E1 might increase cancer risk.
Many researches have been focused on polymorphisms of the CYP2E1. There are many known polymorphic loci in the CYP2E1. Among these polymorphisms, Rsal polymorphism (CYP2E1*5B) (rs2031920) has been associated with higher transcription and increased enzyme activity and is located at 5' regulatory region (Grove et al., 1998; Hayashi et al., 1991; Tsutsumi et al., 1994). Rsal polymorphism is associated with alcoholic liver disease in Asian population (Piao et al., 2003; Tanaka et al., 1997; Tsutsumi et al., 1994). Likewise, some studies show that CYP2E1 polymorphism were associated with risk of HCC (Ladero et al., 1996; Yu et 


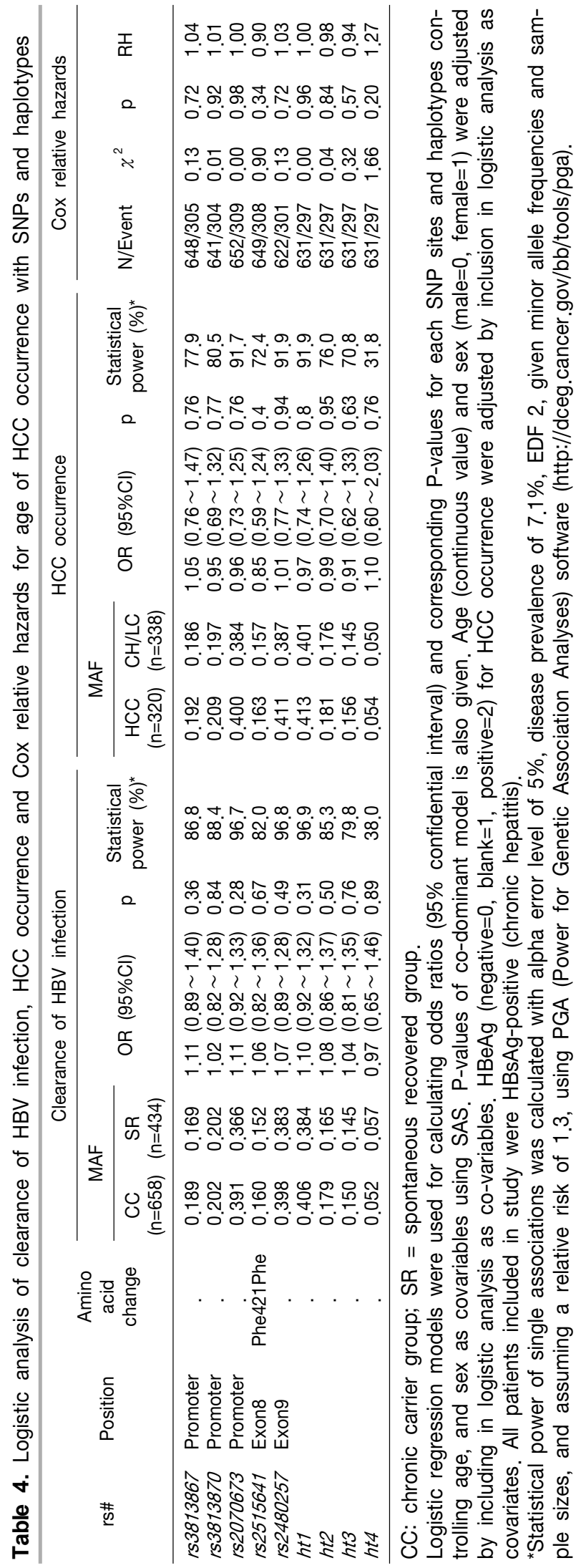

al., 1995). These studies further supports the polymorphism of CYP2E1 can influence to risk of HCC.

In this study, we hypothesized that polymorphisms of CYP2E1 can affect HCC Risk in HBV patient. We analyzed five polymorphisms of CYP2E1 via genotyping a total of 1,092 samples, including 658 chronic carrier and 434 spontaneously recovered individuals. However, our research revealed that the five polymorphisms and haplotypes in CYP2E1 are not associated with HBV clearance, HCC occurrence and onset age of HCC in a Korean population.

Some of the previous studies have shown findings on CYP2E1 polymorphism associations with CYP2E1 activities and HCC risk (Hayashi et al., 1991; Ladero et al., 1996; Lucas et al., 1995; Marchand et al., 1999; Watanabe et al., 1994; Yu et al., 1995). However, other previous studies have reported that there was no association between CYP2E1 genetic polymorphisms and CYP2E1 activities (Carriere et al., 1996; Inoue et al., 2000; Powell et al., 1998). In other words, including the findings of this study, the literature have shown contradictory findings on associations of CYP2E1 polymorphisms with CYP2E1 activities and HCC risk, especially rs3813867 polymorphism which is in absolute linkage disequilibrium (LD) $\left(\left|D^{\prime}\right|=1\right.$ and $\left.r^{2}=1\right)$ with Rsal polymorphism (CYP2E1*5B) (rs2031920), which is associated with higher transcription, increased enzyme activity, and alcoholic liver disease (Grove et al., 1998; Hayashi et al., 1991; Piao et al., 2003; Tanaka et al., 1997; Tsutsumi et al., 1994).

When comparing the contrasting results of previous researches, we can find different statistical methods and patient group in the researches. Positive result studies used a method of classification by physiological determinants, especially drinking and smoking habits. However, negative result studies only performed a case-control analysis without classifying according to alcohol consumption. Moreover, a previous study has shown that the activity of CYP2E1 is modulated by various physiological determinants, such as obesity (O'Shea et al., 1994), fasting (O'Shea et al., 1994), and liver dysfunction (Dilger et al., 1997), and can be induced by ethanol (Girre et al., 1994). In contrast, dietary isothiocyanates (Leclercq et al., 1998), and garlic (Reicks and Crankshaw, 1996; Yang et al., 1994), as well as some drugs, such as disulfiram (Kharasch et al., 1993), and chlormethiazole (Gebhardt et al., 1997), inhibit CYP2E1 activity. Therefore, we can estimate that Rsal polymorphism is not associated with CYP2E1 activity, but rather, is associated with inducement of CYP2E1 activity by gene-environment interactions, including links with ethanol consumption. Moreover, the increase of CYP2E1 activity may be associated with increase of alcoholic liver dis- 
ease risk resulting in increased formation of ROS (Hayashi et al., 1991; Tsutsumi et al., 1994). This means that the findings from previous studies, which suggested that variation of CYP2E1 can influence the risk of HCC, is due to CYP2E1 activity change by ethanol consumption. In other words, we can presume that if the influence of environmental factors would have been be same, then there would be no association signals for the polymorphisms. We can presumption that CYP2E1 polymorphism is associated with CYP2E1 activity induced by ethanol, but is not directly associated with HCC risk. However, we can estimate another hypothesissuggesting that this contradictory result may be affected by racial differences. Additional studies are needed to clarify the association of CYP2E1 with HCC risk in other ethnic populations.

In conclusion, this study presents that even if CYP2E1 may have important functions in the HBV clearance and/or HCC occurrence, genetic variants of CYP2E1 in this study probably do not influence HBV clearance and/or HCC occurrence. Although we could not find any significant associations, the information from this research would be useful for host genetic studies of CYP2E1 related diseases, including $\mathrm{HCC}$. Moreover, CYP2E1 novel polymorphisms, which were identified in our research, will be useful for studies on alcohol related diseases.

\section{Acknowledgments}

We greatly acknowledge and thank the study participants and their families who took part in the HBV cohort study by Seoul National University. This work was supported by a grant from Korea Science and Engineering Foundation (KOSEF) funded by the Korea government (MEST) (No. 2009-0080157) and was also supported by a grant from the National R\&D Program for Cancer Control, Ministry for Health, Welfare and Family affairs, Republic of Korea (0920280).

\section{References}

Baan, R., Straif, K., Grosse, Y., Secretan, B., El Ghissassi, F., Bouvard, V., Altieri, A., and Cogliano, V. (2007). Carcinogenicity of alcoholic beverages. Lancet Oncol. 8, 292-293.

Bae, S.H., Yoon, S.K., Jang, J.W., Kim, C.W., Nam, S.W., Choi, J.Y., Kim, B.S., Park, Y.M., Suzuki, S., Sugauchi, F., and Mizokami, M. (2005). Hepatitis B virus genotype C prevails among chronic carriers of the virus in Korea. J. Korean Med. Sci. 20, 816-820.

Barrett, J.C., Fry, B., Maller, J., and Daly, M.J. (2005). Haploview: analysis and visualization of LD and haplotype maps. Bioinformatics 21, 263-265.
Bosch, F.X., Ribes, J., Cléries, R., and Díaz, M. (2005). Epidemiology of hepatocellular carcinoma. Clin. Liver Dis. 9, 191-211.

Bruix, J., Sherman, M., Llovet, J.M., Beaugrand, M., Lencioni, R., Burroughs, A.K., Christensen, E., Pagliaro, L., Colombo, M., and Rodes, J. (2001). Clinical management of hepatocellular carcinoma. Conclusions of the Barcelona-2000 EASL conference. European Association for the Study of the Liver. J. Hepatol. 35, 421-430.

Carriere, V., Berthou, F., Baird, S., Belloc, C., Beaune, P., and de Waziers, I. (1996). Human cytochrome P450 2E1 (CYP2E1): from genotype to phenotype. Pharmacogenetics 6, 203-211.

Cruz, A.C., Frentzen, B.H., and Behnke, M. (1987). Hepatitis $\mathrm{B}$ : a case for prenatal screening of all patients. $A m$. J. Obstet. Gynecol. 156, 1180-1183.

Dilger, K., Metzler, J., Bode, J.C., and Klotz, U. (1997). CYP2E1 activity in patients with alcoholic liver disease. J. Hepatol. 27, 1009-1014.

El-Serag, H.B., and Mason, A.C. (2000). Risk factors for the rising rates of primary liver cancer in the United States. Arch. Intern. Med. 160, 3227-3230.

Gebhardt, A.C., Lucas, D., Menez, J.F., and Seitz, H.K. (1997). Chlormethiazole inhibition of cytochrome P450 $2 \mathrm{E} 1$ as assessed by chlorzoxazone hydroxylation in humans. Hepatology 26, 957-961.

Girre, C., Lucas, D., Hispard, E., Menez, C., Dally, S., and Menez, J.F. (1994). Assessment of cytochrome P4502E1 induction in alcoholic patients by chlorzoxazone pharmacokinetics. Biochem. Pharmacol. 47, 1503-1508.

Grove, J., Brown, A.S., Daly, A.K., Bassendine, M.F., James, O.F., and Day, C.P. (1998). The Rsal polymorphism of CYP2E1 and susceptibility to alcoholic liver disease in Caucasians: effect on age of presentation and dependence on alcohol dehydrogenase genotype. Pharmacogenetics 8, 335-342.

Guengerich, F.P., Kim, D.H., and Iwasaki, M. (1991). Role of human cytochrome P-450 IIE1 in the oxidation of many low molecular weight cancer suspects. Chem. Res. Toxicol. 4, 168-179.

Hayashi, S., Watanabe, J., and Kawajiri, K. (1991). Genetic polymorphisms in the $5^{\prime}$-flanking region change transcriptional regulation of the human cytochrome P450llE1 gene. J. Biochem. 110, 559-565.

Hedrick, P., and Kumar, S. (2001). Mutation and linkage disequilibrium in human mtDNA. Eur. J. Hum. Genet. 9, 969-972.

Inoue, K., Yamazaki, H., and Shimada, T. (2000). Characterization of liver microsomal 7-ethoxycoumarin O-deethylation and chlorzoxazone 6-hydroxylation activities in Japanese and Caucasian subjects genotyped for CYP2E1 gene. Arch. Toxicol. 74, 372-378.

Kharasch, E.D., Thummel, K.E., Mhyre, J., and Lillibridge, J.H. (1993). Single-dose disulfiram inhibition of chlorzoxazone metabolism: a clinical probe for P450 2E1. Clin. Pharmacol. Ther. 53, 643-650.

Koop, D.R. (1992). Oxidative and reductive metabolism by cytochrome P450 2E1. Faseb. J. 6, 724-730.

Ladero, J.M., Agundez, J.A., Rodriguez-Lescure, A., Diaz- 
Rubio, M., and Benitez, J. (1996). Rsal polymorphism at the cytochrome P4502E1 locus and risk of hepatocellular carcinoma. Gut 39, 330-333.

Lavanchy, D. (2005). Worldwide epidemiology of HBV infection, disease burden, and vaccine prevention. J. Clin. Virol. 34, S1-3.

Leclercq, I., Desager, J.P., and Horsmans, Y. (1998). Inhibition of chlorzoxazone metabolism, a clinical probe for CYP2E1, by a single ingestion of watercress. Clin. Pharmacol. Ther. 64, 144-149.

Lee, M.S., Kim, D.H., Kim, H., Lee, H.S., Kim, C.Y., Park, T.S., Yoo, K.Y., Park, B.J., and Ahn, Y.O. (1998). Hepatitis $B$ vaccination and reduced risk of primary liver cancer among male adults: a cohort study in Korea. Int. J. Epidemiol. 27, 316-319.

Livak, K.J. (1999). Allelic discrimination using fluorogenic probes and the $5^{\prime}$ nuclease assay. Genet. Anal. 14, 143149.

Lucas, D., Menez, C., Girre, C., Berthou, F., Bodenez, P., Joannet, I., Hispard, E., Bardou, L.G., and Menez, J.F. (1995). Cytochrome P450 2E1 genotype and chlorzoxazone metabolism in healthy and alcoholic Caucasian subjects. Pharmacogenetics 5, 298-304.

Marchand, L.L., Wilkinson, G.R., and Wilkens, L.R. (1999). Genetic and dietary predictors of CYP2E1 activity: a phenotyping study in Hawaii Japanese using chlorzoxazone. Cancer Epidemiol. Biomarkers Prev. 8, 495-500.

McCarver, D.G., Byun, R., Hines, R.N., Hichme, M., and Wegenek, W. (1998). A genetic polymorphism in the regulatory sequences of human CYP2E1: association with increased chlorzoxazone hydroxylation in the presence of obesity and ethanol intake. Toxicol. Appl. Pharmacol. 152, 276-281.

Menashe, I., Rosenberg, P.S., and Chen, B.E. (2008). PGA: power calculator for case-control genetic association analyses. BMC Genet 9, 36 .

Morgan, T.R., Mandayam, S., and Jamal, M.M. (2004). Alcohol and hepatocellular carcinoma. Gastroenterology 127, S87-96.

O'Shea, D., Davis, S.N., Kim, R.B., and Wilkinson, G.R. (1994). Effect of fasting and obesity in humans on the 6-hydroxylation of chlorzoxazone: a putative probe of CYP2E1 activity. Clin. Pharmacol. Ther. 56: 359-367.

Odgerel, Z., Nho, K.B., Moon, J.Y., Kee, S.H., Park, K.S., Song, K.J., Baek, L.J., Yeon, J.E., Byun, K.S., Lee, C.H., and Song, J.W. (2003). Complete genome sequence and phylogenetic analysis of hepatitis B virus (HBV) isolates from patients with chronic HBV infection in Korea. J. Med. Virol. 71, 499-503

Peters, T.J., and Ward, R.J. (1988). Role of acetaldehyde in the pathogenesis of alcoholic liver disease. Mol. Aspects Med. 10, 179-190.

Piao, Y.F., Li, J.T., and Shi, Y. (2003). Relationship between genetic polymorphism of cytochrome P450IIE1 and fatty liver. World J. Gastroenterol. 9, 2612-2615.

Powell, H., Kitteringham, N.R., Pirmohamed, M., Smith,
D.A., and Park, B.K. (1998). Expression of cytochrome P4502E1 in human liver: assessment by mRNA, genotype and phenotype. Pharmacogenetics 8, 411-421.

Reicks, M.M., and Crankshaw, D.L. (1996). Modulation of rat hepatic cytochrome P-450 activity by garlic organosulfur compounds. Nutr. Cancer 25, 241-248.

Seitz, H.K., and Stickel, F. (2007). Molecular mechanisms of alcohol-mediated carcinogenesis. Nat. Rev. Cancer 7, 599-612.

Song, B.C., Cui, X.J., and Kim, H. (2005a). Hepatitis B virus genotypes in Korea: an endemic area of hepatitis $B$ virus infection. Intervirology 48, 133-137.

Song, B.C., Kim, H., Kim, S.H., Cha, C.Y., Kook, Y.H., and Kim, B.J. (2005b). Comparison of full length sequences of hepatitis B virus isolates in hepatocellular carcinoma patients and asymptomatic carriers of Korea. J. Med. Virol. 75, 13-19.

Stephens, M., Smith, N.J., and Donnelly, P. (2001). A new statistical method for haplotype reconstruction from population data. Am. J. Hum. Genet. 68, 978-989.

Stickel, F., and Osterreicher, C.H. (2006). The role of genetic polymorphisms in alcoholic liver disease. Alcohol Alcohol 41, 209-224.

Stickel, F., Schuppan, D., Hahn, E.G., and Seitz, H.K. (2002). Cocarcinogenic effects of alcohol in hepatocarcinogenesis. Gut 51, 132-139.

Takahashi, T., Lasker, J.M., Rosman, A.S., and Lieber, C.S. (1993). Induction of cytochrome P-4502E1 in the human liver by ethanol is caused by a corresponding increase in encoding messenger RNA. Hepatology 17, 236-245.

Tanaka, F., Shiratori, Y., Yokosuka, O., Imazeki, F., Tsukada, Y., and Omata, M. (1997). Polymorphism of alcohol-metabolizing genes affects drinking behavior and alcoholic liver disease in Japanese men. Alcohol Clin. Exp. Res. 21, 596-601.

Tsutsumi, M., Takada, A., and Wang, J.S. (1994). Genetic polymorphisms of cytochrome P4502E1 related to the development of alcoholic liver disease. Gastroenterology 107, 1430-1435.

Watanabe, J., Hayashi, S., and Kawajiri, K. (1994). Different regulation and expression of the human CYP2E1 gene due to the Rsal polymorphism in the $5^{\prime}$-flanking region. J. Biochem. 116, 321-326.

Yang, C.S., Smith, T.J., and Hong, J.Y. (1994). Cytochrome $\mathrm{P}-450$ enzymes as targets for chemoprevention against chemical carcinogenesis and toxicity: opportunities and limitations. Cancer Res. 54, 1982s-1986s.

Yoo, B.C., Park, J.W., Kim, H.J., Lee, D.H., Cha, Y.J., and Park, S.M. (2003). Precore and core promoter mutations of hepatitis $B$ virus and hepatitis $B$ e antigen-negative chronic hepatitis B in Korea. J. Hepatol. 38, 98-103.

Yu, M.W., Gladek-Yarborough, A., Chiamprasert, S., Santella, R.M., Liaw, Y.F., and Chen, C.J. (1995). Cytochrome P450 2E1 and glutathione S-transferase M1 polymorphisms and susceptibility to hepatocellular carcinoma. Gastroenterology 109, 1266-1273. 